\title{
SORÇÃO DO HERBICIDA ACETOCHLOR EM AMOSTRAS DE SOLO, ÁCIDOS HÚMICOS E HUMINAS DE ARGISSOLO SUBMETIDO À SEMEADURA DIRETA E AO PREPARO CONVENCIONAL ${ }^{(1)}$
}

\author{
Miguel Vicente Weiss Ferri ${ }^{(2)}$, Juliana Gomes ${ }^{(3)}$, Deborah Pinheiro \\ Dick $^{(4)}$, Roberto Fernando de Souza ${ }^{(4)}$ \& Ribas Antonio Vidal ${ }^{(5)}$
}

\begin{abstract}
RESUMO
A sorção de herbicidas no solo é um dos processos determinantes na sua dinâmica no ambiente. Para compostos fracamente polares, como é caso do acetochlor, a matéria orgânica do solo constitui o principal sorvente. $O$ objetivo deste estudo foi avaliar a sorção de acetochlor em amostras de solo, de ácidos húmicos e de huminas de um Argissolo Vermelho distrófico (PVd) submetido à semeadura direta e ao preparo convencional. Isotermas de sorção foram obtidas em temperatura ambiente e a concentração do herbicida foi determinada por cromatografia líquida de alta eficiência. As amostras de solo foram caracterizadas pelos teores de C orgânico e de substâncias húmicas; os ácidos húmicos e huminas foram caracterizados por análise elementar. A capacidade de sorção de acetochlor foi superior no solo de semeadura direta $\left(K_{d}=1,22 \pm\right.$ $\left.0,11 \mathrm{~L} \mathrm{~kg}^{-1}, \mathrm{~K}_{\mathrm{OC}}=116 \pm 10 \mathrm{~L} \mathrm{~kg}^{-1} \mathrm{C}\right)$ em relação ao preparo convencional $\left(K_{d}=0,76 \pm 0,08 \mathrm{~L} \mathrm{~kg}^{-1}, \mathrm{~K}_{\mathrm{OC}}=86 \pm 8 \mathrm{~L} \mathrm{~kg}^{-1} \mathrm{C}\right)$. Este comportamento foi relacionado, em parte, com o maior teor de $\mathrm{C}$ no solo tratado com semeadura direta. Nos ácidos húmicos de preparo convencional, a sorção $\left(K_{d}=178 \pm 18,9 \mathrm{~L} \mathrm{~kg}^{-1}\right.$, $\mathrm{K}_{\mathrm{OC}}=352 \pm 37 \mathrm{~L} \mathrm{~kg}^{-1} \mathrm{C}$ ) foi similar à verificada nos ácidos húmicos de semeadura direta $\left(K_{d}=158 \pm 14,6 \mathrm{~L} \mathrm{~kg}^{-1}, \mathrm{~K}_{\mathrm{OC}}=321 \pm 30 \mathrm{~L} \mathrm{~kg}^{-1} \mathrm{C}\right)$; situação semelhante foi observada com as huminas. Dentre as frações húmicas avaliadas, as huminas apresentaram maior capacidade de sorção $\left(\mathrm{Kd}=1.028\right.$ e $1.183 \mathrm{~L} \mathrm{~kg}^{-1}, \mathrm{~K}_{\mathrm{OC}}=2.691$ e $\left.2.892 \mathrm{~L} \mathrm{~kg}^{-1} \mathrm{C}\right)$.
\end{abstract}

Termos de indexação: substâncias húmicas, manejo de solos, cromatografia líquida, matéria orgânica.

(1) Parte da Tese de Doutorado do primeiro autor, apresentada à Faculdade de Agronomia da Universidade Federal do Rio Grande do Sul - UFRGS. Recebido para publicação em janeiro de 2003 e aprovado em agosto de 2005.

(2) Engenheiro-Agrônomo do Instituto de Química, Universidade Federal do Rio Grande do Sul - UFRGS. Av. Bento Gonçalves 9500, CEP 91501-970 Porto Alegre (RS). Bolsista FAPERGS. E-mail: mvwferri@bol.com.br

(3) Doutoranda do Programa de Pós-graduação em Ciência do Solo da Faculdade de Agronomia, UFRGS. E-mail: julianag@cpovo.net

(4) Professora adjunta, Departamento de Físico-Química, Instituto de Química, UFRGS. E-mail: dpdick@iq.ufrgs.br

(5) Professor do Departamento de Plantas de Lavoura, Faculdade de Agronomia, UFRGS. E-mail: ribas.vidal@ufrgs.br 


\title{
SUMMARY: SORPTION OF ACETOCHLOR HERBICIDE BY SOIL SAMPLES, HUMIC ACIDS AND HUMIN FROM AN ARGISOL UNDER NO-TILL AND CONVENTIONAL TILLAGE SYSTEMS
}

\begin{abstract}
The sorption of herbicides to soil is a process determinant of its dynamics in the environment. For weakly polar compounds such as acetochlor, organic matter represents the main sorbent in soil. The goal of this study was to evaluate the sorption of acetochlor on soil samples, humic acids and humin from an "Argissolo Vermelho distrófico" (PVdPaleudult) under no-till and conventional tillage systems. Sorption isotherms were carried out at room temperature and the herbicide concentration was determined by high performance liquid chromatography. The samples were characterized by their contents of organic carbon and of humic substances; the humic acids and humin were characterized by elemental analyses. The sorption capacity of acetochlor was higher in the no-till soil $\left(K_{d}=1.22 \pm\right.$ $\left.0.11 \mathrm{~L} \mathrm{~kg}^{-1}, K_{O C}=116 \pm 10 \mathrm{~L} \mathrm{~kg}^{-1} \mathrm{C}\right)$ in comparison to that under conventional tillage $\left(K_{d}=\right.$ $\left.0.76 \pm 0.08 \mathrm{~L} \mathrm{~kg}^{-1}, K_{O C}=86 \pm 8 \mathrm{~L} \mathrm{~kg}^{-1} \mathrm{C}\right)$. This behavior was partially related to the higher organic carbon content of the no-till sample. Sorption by humic acids from conventional tillage soil $\left(K_{d}=178 \pm 18.9 \mathrm{~L} \mathrm{~kg}^{-1}, K_{O C}=352 \mathrm{~L} \mathrm{~kg}^{-1} \mathrm{C}\right)$ was similar to that from the no-till soil ( $\left.K_{d}=158 \pm 14.6 \mathrm{~L} \mathrm{~kg}^{-1}, K_{O C}=321 \pm 30 \mathrm{~L} \mathrm{~kg}^{-1} \mathrm{C}\right)$; a similar situation was verified with humin. Among the analyzed humic fractions, humin presented the highest sorption capacity $\left(\mathrm{Kd}=1.028\right.$ and $1.183 \mathrm{~L} \mathrm{~kg}^{-1}, K_{O C}=2.691$ and $\left.2.892 \mathrm{~L} \mathrm{~kg}^{-1} \mathrm{C}\right)$.
\end{abstract}

Index terms: humic substances, soil management, liquid chromatography, organic matter.

\section{INTRODUÇÃO}

A sorção de herbicidas no solo afeta a sua absorção pelas raízes de plantas daninhas e os processos de sua dissipação, tais como: degradação biológica, volatilização, lixiviação e transporte por erosão hídrica (Herwig et al., 2001). Conseqüentemente, a eficácia de controle das plantas daninhas e o risco de contaminação ambiental por estes compostos também são afetados. O tipo e conteúdo de argila, teor e características da matéria orgânica (MO) e umidade do solo afetam as interações do herbicida no solo (Levanon et al., 1993; Czapar et al., 1994). A MO apresenta acentuada capacidade de sorver herbicidas (Stevenson, 1972) e isto reduz a atividade biológica e a mobilidade dos compostos químicos aplicados ao solo (Lee \& Farmer, 1989; Scheunert et al., 1992). A pronunciada reatividade da MO está relacionada principalmente com sua elevada área superficial específica e presença de vários grupos funcionais, como carboxilas, hidroxilas e aminas, e estruturas alifáticas e aromáticas (Stevenson, 1972; Stearman et al., 1989; Kuckuk et al., 1997).

O herbicida acetochlor [2-cloro-n-(etoximetil)-n(2-etil-6-metil-fenil)acetamida], pertencente ao grupo químico das cloroacetamidas, é utilizado, no Brasil, no controle de gramíneas nas culturas de milho, soja e cana-de-açúcar (Vidal \& Fleck, 2001). Este composto constitui uma das três opções de ingredientes ativos de herbicidas, e, no País, aproximadamente 4 milhões de hectares sob cultura de milho recebem aplicação de herbicidas (Nakamae, 2002). O acetochlor é fracamente polar, apresenta moderada solubilidade em água $\left(223 \mathrm{~g} \mathrm{~L}^{-1}\right)$, baixa pressão de vapor $\left(3,4 \times 10^{-8} \mathrm{~mm} \mathrm{Hg}\right)$, elevada partição em meio orgânico $\left(\mathrm{Kow}=300\left(\mathrm{~mol} \mathrm{~L}^{-1}\right.\right.$ octanol) / (mol L-1 água)) e sua atividade independe do $\mathrm{pH}$ do meio (Vidal \& Fleck, 2001). Os herbicidas cloroacetamidas têm sido usados largamente desde 1980, destacando-se também os compostos alachlor e metolachlor (Crisanto et al., 1995).

A equação da isoterma linear, que permite o cálculo do coeficiente de partição $K_{d}$ e de partição em relação ao teor de $\mathrm{C}$ orgânico $\mathrm{K}_{\mathrm{OC}}$, tem sido empregada freqüentemente para estudar a sorção de compostos orgânicos antropogênicos em solos e sedimentos (Rutheford et al., 1992; Kile et al., 1995). $\mathrm{O}$ aumento na sorção de herbicidas cloroacetamidas no solo com o incremento no teor de MO é reportado na literatura por Weber \& Peter (1982), Wood et al. (1987) e Crisanto et al. (1995), dentre outros.

A sorção de metolachlor depende do teor de C orgânico $\left(\mathrm{K}_{\mathrm{OC}}\right)$ e varia de 28 a $663 \mathrm{~L} \mathrm{~kg}^{-1}$, conforme o solo avaliado (Pusino et al., 1992). A interação dos herbicidas cloroacetamidas e a MO pode ocorrer não só por meio de pontes de $\mathrm{H}$ entre grupamentos amina e carbonílico das moléculas destes compostos e grupos carboxílicos e hidroxílicos das superfícies orgânicas, mas também por ligação de transferência de carga $(\pi)$ entre núcleos aromáticos dos herbicidas e da MO humificada do solo (Peter \& Weber, 1985; Senesi et al., 1994). Senesi et al. (1994), ao estudarem a sorção do herbicida alachlor em ácidos húmicos, constataram que interações hidrofóbicas também contribuem para a retenção de herbicidas cloroacetamidas. 
A MO do solo pode ser fracionada quimicamente em ácidos húmicos, ácidos fúlvicos e huminas, que se diferenciam quanto à composição química e estrutura molecular e, portanto, apresentam diferentes reatividades (Stevenson, 1994). A proporção das frações húmicas e as características químicas e moleculares da MO são influenciadas principalmente pelo ambiente pedogênico e pelo uso do solo (Torrents et al., 1997; Empinotti, 1999; Doane et al., 2003).

Sistemas de manejo de solo afetam diferentemente o teor e a qualidade da MO do solo (Bayer \& Bertol, 1999), bem como a proporção de substâncias húmicas (Empinotti, 1999). Ácidos húmicos extraídos de Argissolo sob semeadura direta apresentaram menor grau de humificação, inferido pelo menor teor de radicais semiquinonas na sua estrutura, do que amostras extraídas de solo sob preparo convencional (Bayer et al., 2002).

Ao analisar as diferenças existentes entre a MO oriunda de sistemas de semeadura direta (10 anos consecutivos) e de preparo convencional, Arshad et al. (1990) observaram maior quantidade de estruturas alifáticas na MO oriunda da semeadura direta, quando comparada à do preparo convencional. A sorção de acetochlor em Argissolo Vermelho foi superior nas amostras sob plantio direto, quando comparada ao do preparo convencional (Ferri et al., 2002). Este comportamento diferenciado não foi explicado pela composição elementar da MO presente no solo, mas foi atribuído a possíveis diferenças no arranjo estrutural da molécula de MO e outras características químicas do solo.

Considerando que o tipo de manejo do solo pode influir no teor e características da MO e das substâncias húmicas, diferenças na sorção de herbicidas são esperadas em solos submetidos a diferentes sistemas de manejo.

Este trabalho teve por objetivo investigar o potencial de sorção do herbicida acetochlor em amostras de solo, de ácidos húmicos e de huminas, oriundas de Argissolo Vermelho distrófico submetido à semeadura direta e ao preparo convencional.

\section{MATERIAL E MÉTODOS}

\section{Local do experimento e coleta das amostras}

O estudo foi realizado na Estação Experimental Agronômica da Universidade Federal do Rio Grande do Sul (EEA/UFRGS), localizada no município de Eldorado do Sul (RS). Utilizou-se um Argissolo Vermelho distrófico típico (Embrapa, 1999) de textura franco-argilosa, com $220 \mathrm{~g} \mathrm{~kg}^{-1}$ de argila, $240 \mathrm{~g} \mathrm{~kg}^{-1}$ de silte e $540 \mathrm{~g} \mathrm{~kg}^{-1}$ de areia.
O experimento consistiu em dois sistemas de preparo: semeadura direta (cinco anos consecutivos) e preparo convencional (cinco anos, aração seguida de cinco gradagens num ano), sendo cultivada em ambas as áreas a sucessão aveia preta-milho. Antes da instalação do experimento, a área encontrava-se sob pastagem nativa, apresentando, na camada de 0-10 cm, teor de C orgânico de 19,3 $\mathrm{g} \mathrm{kg}^{-1}, \mathrm{pH}$ de 4,7 e CTC de $90 \mathrm{mmol}_{\mathrm{c}} \mathrm{kg}^{-1}$. O delineamento experimental utilizado foi de blocos casualizados com quatro repetições, apresentando cada unidade experimental dimensões de $2 \times 10 \mathrm{~m}$. Para cada sistema de manejo foram coletadas 20 subamostras da camada de $0-10 \mathrm{~cm}$, imediatamente antes da semeadura da cultura do milho, utilizando trado cilíndrico com $5 \mathrm{~cm}$ de diâmetro, constituindo uma amostra composta para cada tratamento. Após a coleta, as amostras de solo foram secas ao ar em temperatura ambiente, moídas e passadas em peneira com malha de $2 \mathrm{~mm}$. O pH das amostras de solo foi de 5,4, na semeadura direta, e de 5,5, no preparo convencional, medido em água destilada e usando a relação 1:2,5 solo:água, em duas repetições.

\section{Fracionamento e quantificação das substâncias húmicas}

As substâncias húmicas foram fracionadas quimicamente, segundo método de Dick et al. (1998), adaptado de Swift (1996). Foram realizadas três repetições para cada solo. A $3 \mathrm{~g}$ de solo foram adicionados $30 \mathrm{~mL}$ de $\mathrm{HCl} 0,5 \mathrm{~mol} \mathrm{~L}^{-1}$ e a suspensão foi agitada por $2 \mathrm{~h}$. Após centrifugação (4.000 rpm, $10 \mathrm{~min}$ ), o sobrenadante foi separado e armazenado. Este procedimento foi repetido três vezes, obtendose, nesta etapa, o extrato ácido que continha MO de baixo peso molecular. Em seguida, o solo foi submetido a tratamento com $30 \mathrm{~mL}$ de $\mathrm{NaOH} 0,5 \mathrm{~mol} \mathrm{~L}^{-1} \mathrm{sob}$ agitação de $3 \mathrm{~h}$. O extrato alcalino foi separado por centrifugação (4.000 rpm, $10 \mathrm{~min}$ ). Este procedimento foi repetido quatro vezes. $\mathrm{O}$ volume total do extrato alcalino foi medido, uma alíquota para análise do teor de $\mathrm{C}$ correspondente às substâncias húmicas solúveis $\left(\mathrm{SH}_{\mathrm{S}}\right)$ foi retirada, e a solução foi acidificada a pH 2,0 com $\mathrm{HCl} 0,1 \mathrm{~mol} \mathrm{~L}^{-1}$. Após $24 \mathrm{~h}$ de repouso, a fração de ácidos fúlvicos (AF) (sobrenadante) foi separada por centrifugação. $\mathrm{O}$ volume do extrato de ácidos fúlvicos foi medido, e uma alíquota foi retirada para análise do teor de $\mathrm{C}$ orgânico.

Os teores de $\mathrm{C}$ no extrato ácido $\left(\mathrm{C}_{\mathrm{HCl}}\right)$, no extrato das substâncias húmicas solúveis $\left(\mathrm{C}_{\mathrm{SHS}}\right)$ e no extrato de ácidos fúlvicos $\left(\mathrm{C}_{\mathrm{AF}}\right)$, foram quantificados, determinando-se a absorbância a $580 \mathrm{~nm}$ após oxidação da MO com dicromato de potássio em meio ácido a $60{ }^{\circ} \mathrm{C}$, durante $4 \mathrm{~h}$ (Dick et al., 1998). As quantidades de MO de baixo peso molecular, de substâncias húmicas solúveis e de ácidos fúlvicos foram estimadas pelos valores obtidos em $\mathrm{C}_{\mathrm{HCl}}, \mathrm{C}_{\mathrm{SHS}}$ e $\mathrm{C}_{\mathrm{AF}}$, considerando que, nos respectivos extratos, estas são as únicas formas presentes de $\mathrm{C}$ (Dick et al., 1998). O teor de $\mathrm{C}$ devido aos ácidos húmicos foi 
inferido a partir da equação: $\mathrm{C}_{\mathrm{AH}}=\mathrm{C}_{\mathrm{SHS}}-\mathrm{C}_{\mathrm{AF}}$, em que $\mathrm{C}_{\mathrm{AH}}$ representa o teor de $\mathrm{C}$ sob a forma de ácidos húmicos. Para obter o teor de $\mathrm{C}$ presente na forma de huminas $\left(\mathrm{C}_{\mathrm{HU}}\right)$, empregou-se a fórmula: $\mathrm{C}_{\mathrm{HU}}=$ $\mathrm{C}_{\mathrm{T}}-\left(\mathrm{C}_{\mathrm{SHS}}+\mathrm{C}_{\mathrm{HCl}}\right)$, em que $\mathrm{C}_{\mathrm{T}}$ é o teor de $\mathrm{C}$ total.

Para as reações de sorção, os ácidos húmicos foram purificados com $30 \mathrm{~mL}$ de solução de $\mathrm{HF} / \mathrm{HCl}$ $(5 / 5 \% \mathrm{v} / \mathrm{v})$ sob agitação por $1 \mathrm{~h}$, repetindo-se o procedimento quatro vezes (Dick et al., 1998). Após lavagem com água destilada (três vezes), a fração de ácidos húmicos foi congelada (16 h), descongelada, centrifugada e seca em estufa a vácuo a $60{ }^{\circ} \mathrm{C}$. A fração huminas foi obtida tratando-se o resíduo de solo remanescente do tratamento alcalino com $30 \mathrm{~mL}$ de solução de HF a $10 \%$ (v/v), sob agitação por $2 \mathrm{~h}$, segundo método descrito em Schmidt et al. (1997). Este procedimento foi repetido dez vezes, e a fração concentrada de huminas foi lavada cinco vezes com água destilada e seca em estufa a vácuo a $60{ }^{\circ} \mathrm{C}$.

\section{Caracterização dos sorventes}

A composição elementar de ácidos húmicos e de huminas foi determinada em analisador elementar Perkin Elmer 2400 em duplicata. Os teores de C, H e $\mathrm{N}$ foram corrigidos para uma base livre de cinzas, determinada gravimetricamente por calcinação de amostras de ácidos húmicos e huminas a $750{ }^{\circ} \mathrm{C}$, durante $4 \mathrm{~h}$. O teor de oxigênio foi calculado por diferença: $\mathrm{O}\left(\mathrm{mg} \mathrm{g}^{-1}\right)=1.000-\left(\mathrm{C}+\mathrm{H}+\mathrm{N} \mathrm{mg} \mathrm{g}{ }^{-1}\right)$. Foram calculadas as razões atômicas $\mathrm{H} / \mathrm{C}$ e O/C e a relação $\mathrm{C} / \mathrm{N}$.

A identificação da fração mineral nas huminas após tratamento com solução de $\mathrm{HF}$ foi realizada em lâmina em pó por difratometria de raios X (DRX) (difratômetro Siemens, modelo Kristalloflex D 5000), empregando-se tubo de radiação de $\mathrm{Cu}$ com comprimento de onda de 1,5422 $\AA$, voltagem de $40 \mathrm{kV}$ e corrente de $30 \mathrm{~mA}$. A amplitude de leitura foi de 2 a $70^{\circ} 2 \theta$ e a velocidade de varredura foi de $0,025^{\circ} 2 \theta$ por $1,5 \mathrm{~s}$

$\mathrm{O}$ teor de $\mathrm{C}$ orgânico total das amostras de solo foi determinado pelo método Walkley-Black $(\mathrm{n}=3)$, descrito por Tedesco et al. (1995). A área superficial específica (ASE) de amostras de solo foi determinada com três repetições pelo método da adsorção de água (Quirk, 1955), descrito em Gomes et al. (2002).

\section{Isotermas de sorção}

As reações de sorção foram efetuadas em duplicata, agitando-se, por $24 \mathrm{~h}$, em temperatura ambiente, $10 \mathrm{mg}$ de ácidos húmicos ou $30 \mathrm{mg}$ de huminas em $10 \mathrm{~mL}$ de solução que continha 2,5 , $10,15,20,25,30$ e $40 \mathrm{mg} \mathrm{L}^{-1}$ do herbicida acetochlor (99,9\% de pureza) ou $2 \mathrm{~g}$ de solo em $20 \mathrm{~mL}$ de solução de herbicida, nas concentrações anteriormente citadas. A força iônica da solução foi ajustada a $0,01 \mathrm{~mol} \mathrm{~L}^{-1} \mathrm{com} \mathrm{CaCl}_{2}$. Considerando a camada superficial de $1 \mathrm{~cm}$ de solo como sendo a profundidade onde ocorre preferencialmente a sorção de herbicida aplicado, a concentração de acetochlor de $4,2 \mathrm{mg} \mathrm{L}^{-1}$ na relação solo:solução empregada (1:10) no presente estudo corresponderia à dose recomendada em campo (4,2 kg ha $\left.{ }^{-1}\right)$ (Ferri et al., 2002). Após a reação, as amostras foram centrifugadas, filtradas e os sobrenadantes armazenados a $3{ }^{\circ} \mathrm{C}$. $\mathrm{O} \mathrm{pH}$ das soluções com acetochlor foi monitorado antes e depois da sorção do herbicida, variando os valores em torno de 5,6 e 5,5 , respectivamente. Duas provas em branco foram preparadas: a primeira, uma solução de acetochlor na concentração de $40 \mathrm{mg} \mathrm{L}^{-1}$ sem sorvente, para verificar possível degradação do herbicida durante o procedimento de sorção, e a segunda, uma solução de $\mathrm{CaCl}_{2}$ a 0,01 mol L-1 com solo, ácidos húmicos ou huminas sem o herbicida, para verificar a eventual presença de substâncias orgânicas com pico cromatográfico com tempo de retenção semelhante ao herbicida acetochlor. Os resultados destes testes mostraram que o herbicida analisado não se degradou durante o tempo e as condições experimentais empregados na determinação das isotermas, e que não foram co-extraídas substâncias interferentes nas determinações cromatográficas.

As concentrações de herbicida foram determinadas por cromatografia líquida de alta eficiência, utilizando-se cromatógrafo Shimadzu, LC - 10AD, nas seguintes condições cromatográficas: detector ultra-violeta a $220 \mathrm{~nm}$; coluna de fase reversa $\mathrm{C}_{18}$ $250 \times 4,6 \mathrm{~mm}$ (Supelco); temperatura da coluna de $3{ }^{\circ} \mathrm{C}$; fase móvel metanol:água 70:30 (v/v); taxa de fluxo de $0,9 \mathrm{~mL} \mathrm{~min}^{-1}$ e volume de injeção da amostra de $50 \mu \mathrm{L}$. No momento da injeção, as amostras foram filtradas em membranas de $0,45 \mu \mathrm{m}$ de diâmetro de poro. Para cada amostra, foram realizadas duas leituras cromatográficas, e a concentração de acetochlor na solução de equilíbrio foi obtida pela média aritmética dos dois valores. A quantidade sorvida de acetochlor foi obtida pela equação:

$$
\mathrm{Q}_{s}=\frac{\left(\mathrm{C}_{\mathrm{i}}-\mathrm{C}_{\mathrm{oq}}\right) \mathrm{V}}{\mathrm{m}}
$$

em que

$\mathrm{Q}_{\mathrm{S}}\left(\mathrm{mg} \mathrm{kg}^{-1}\right)=$ quantidade de acetochlor sorvido por unidade de massa de solo; $\mathrm{C}_{\mathrm{i}}\left(\mathrm{mg} \mathrm{L}^{-1}\right)=$ concentração inicial de acetochlor; $\mathrm{C}_{\mathrm{eq}}\left(\mathrm{mg} \mathrm{L}^{-1}\right)=$ concentração de acetochlor na solução de equilíbrio;

$\mathrm{V}(\mathrm{L})$ = volume da solução de herbicida; $\mathrm{m}(\mathrm{kg})=$ massa de solo.

Os dados experimentais de quantidade sorvida $\left(\mathrm{Q}_{\mathrm{s}}\right)$ e de concentração de equilíbrio $\left(\mathrm{C}_{\mathrm{eq}}\right)$ obtidos foram ajustados à isoterma:

sendo

$$
\mathrm{Q}_{\mathrm{s}}=\mathrm{a}+\mathrm{K}_{\mathrm{d}} \mathrm{C}_{\mathrm{eq}}
$$

$\mathrm{K}_{\mathrm{d}}\left(\mathrm{L} \mathrm{kg}^{-1}\right)=$ coeficiente de partição do herbicida acetochlor no sorvente;

$\mathrm{a}=$ constante. 
O coeficiente de partição em relação ao teor de C orgânico do sorvente $\left(\mathrm{K}_{\mathrm{OC}}, \mathrm{L} \mathrm{kg}^{-1} \mathrm{C}\right)$ foi calculado pela equação:

$$
\mathrm{K}_{\mathrm{OC}}=\left(\mathrm{K}_{\mathrm{d}} / \mathrm{C}\right) \times 1.000
$$

em que $\mathrm{C}$ representa o teor de $\mathrm{C}$ orgânico do sorvente $\left(\mathrm{g} \mathrm{kg}^{-1}\right)$.

\section{Tratamento estatístico}

Os dados relativos à distribuição de $\mathrm{C}$ no solo e área superficial específica do solo foram submetidos à análise de variância. O efeito qualitativo do preparo do solo foi avaliado pelo teste de Tukey $(\mathrm{P} \leq 0,05)$. Nos testes de sorção do herbicida acetochlor, a qualidade do ajuste linear entre quantidade sorvida e concentração em equilíbrio foi avaliada pelo coeficiente de determinação $\left(R^{2}\right)$ calculado em cada equação a $1 \%$ de significância. Os coeficientes $K_{d}$ e $K_{O C}$ foram comparados pelo erro-padrão da média.

\section{RESULTADOS E DISCUSSÃO}

\section{Teor e qualidade da matéria orgânica e substâncias húmicas}

O manejo do solo influiu no teor de $\mathrm{C}$ e de algumas das frações orgânicas que compunham a MO no solo (Quadro 1). O maior teor de C orgânico total verificado na semeadura direta refletiu-se no maior teor de $\mathrm{MO}$ de baixo peso molecular (extrato de $\mathrm{HCl}$ ) e de ácidos fúlvicos, comparativamente ao do preparo convencional. Provavelmente, estas frações orgânicas de baixo a médio peso molecular e de caráter mais hidrofílico (Stevenson, 1994) permaneceram no solo sob semeadura direta em virtude do não-revolvimento do solo e da manutenção da cobertura na superfície. Teores mais elevados de $\mathrm{C}$ no extrato ácido e no extrato de ácidos fúlvicos de amostras de solo de semeadura direta também foram observados por Empinotti (1999) para Argissolo Vermelho submetido aos mesmos sistemas de manejo do presente estudo durante 13 anos, na rotação das culturas de aveia e de milho. A autora atribuiu este comportamento à mineralização preferencial de ácidos fúlvicos e de compostos orgânicos de baixo peso molecular em sistemas de preparo convencional.

Em ambos os tratamentos, o C orgânico ocorreu principalmente na forma de huminas (48 a $49 \%$ ), seguido de ácidos húmicos (20 a $24 \%$ ), ácidos fúlvicos (18 a 20 \%) e MO de baixo peso molecular (10 a $11 \%$ ).

A composição elementar da fração de ácidos húmicos foi relativamente semelhante em ambos os sistemas de manejo do solo, destacando-se apenas o teor de $\mathrm{C}$ que foi mais elevado na amostra oriunda de plantio convencional (Quadro 2).

Em contrapartida, a fração de huminas da semeadura direta apresentou teores de $\mathrm{H}$ e $\mathrm{C}$ inferiores e teores de $\mathrm{O}$ e $\mathrm{N}$ superiores aos verificados para a amostra de preparo convencional. Os menores valores de razão $\mathrm{H} / \mathrm{C}$ e relação $\mathrm{C} / \mathrm{N}$ e o maior valor de razão $\mathrm{O} / \mathrm{C}$, calculado para as huminas de semeadura direta (Quadro 2), indicam que estas apresentam maior grau de condensação estrutural e maior acúmulo de $\mathrm{N}$ e $\mathrm{O}$ em sua molécula, quando comparadas às huminas de preparo convencional.

Em ambos os sistemas de manejo, as huminas apresentaram caráter mais alifático e maior grau de oxidação (indicados pelos valores superiores de $\mathrm{H} / \mathrm{C}$ e $\mathrm{O} / \mathrm{C}$, respectivamente) do que a fração de ácidos húmicos. Dentre as frações húmicas analisadas, as huminas foram as mais pobres em $\mathrm{N}$.

Em amostra de horizonte A de Chernossolo brasileiro, uma maior proporção de grupamentos alifáticos e maior grau de oxigenação em huminas (indicados pelas maiores razões $\mathrm{H} / \mathrm{C} \mathrm{e} \mathrm{O} / \mathrm{C}$ ), quando comparadas com os respectivos ácidos húmicos, foram corroborados pela análise dos espectros de infra-vermelho obtidos nas duas frações húmicas (Dick et al., 2003).

Quadro 1. Teores de $\mathrm{C}$ orgânico total $\left(\mathrm{C}_{\mathrm{T}}\right)$, de $\mathrm{C}$ presente no extrato ácido $\left(\mathrm{C}_{\mathrm{HCl}}\right)$, de $\mathrm{C}$ sob a forma de substâncias húmicas solúveis $\left(\mathrm{C}_{\mathrm{SHS}}\right)$, de ácidos húmicos $\left(\mathrm{C}_{\mathrm{AH}}\right)$, de ácidos fúlvicos $\left(\mathrm{C}_{\mathrm{AF}}\right)$ e de huminas $\left(\mathrm{C}_{\mathrm{HU}}\right)$ e área superficial específica (ASE) de Argissolo Vermelho submetido à semeadura direta e ao preparo convencional

\begin{tabular}{|c|c|c|c|c|c|c|c|}
\hline Preparodo solo & $\mathbf{C}_{\mathbf{T}}$ & $\mathrm{C}_{\mathrm{H}} \mathrm{Cl}$ & Cshs & $\mathrm{C}_{\text {AH }}$ & $\mathrm{C}_{\text {A F }}$ & $\mathbf{C}_{\mathbf{H U}}$ & ASE \\
\hline & & & 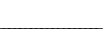 & & & - & $\mathrm{m}^{2} \mathrm{~g}^{-1}$ \\
\hline Semeadura direta & $10,5 \mathrm{a}$ & $1,2 \mathrm{a}$ & $4,2 \mathrm{a}$ & $2,1 \mathrm{a}$ & $2,1 \mathrm{a}$ & $5,1 \mathrm{a}$ & $23,4 \mathrm{a}$ \\
\hline Preparo convencional & $8,8 \mathrm{~b}$ & $0,9 \mathrm{~b}$ & 3,7 a & 2,1 a & $1,6 \mathrm{~b}$ & $4,2 \mathrm{a}$ & $22,1 \mathrm{a}$ \\
\hline $\mathrm{CV}(\%)$ & 6,3 & 12,3 & 13,5 & 17,6 & 11,6 & 15,5 & 8,1 \\
\hline
\end{tabular}

Médias seguidas pela mesma letra, na coluna, não diferem pelo teste de Tukey $(\mathrm{P}>0,05)$. 
Quadro 2. Composição elementar, razões atômicas $\mathrm{O} / \mathrm{C}$ e H/C, relação $\mathrm{C} / \mathrm{N}$ e teor de cinzas dos ácidos húmicos (AH) e de huminas (HU) oriundos de Argissolo Vermelho submetido à semeadura direta (SD) e preparo convencional (PC)

\begin{tabular}{|c|c|c|c|c|c|c|c|c|c|}
\hline \multirow{2}{*}{\multicolumn{2}{|c|}{ Amostra }} & \multicolumn{4}{|c|}{ Composição elementar(1)(2) } & \multicolumn{2}{|c|}{ Razão atômica } & \multirow{2}{*}{ Relação C/N } & \multirow{2}{*}{ Cinza } \\
\hline & & C & $\mathbf{H}$ & $\mathbf{O}^{(3)}$ & $\mathbf{N}$ & $\mathrm{O} / \mathrm{C}$ & $\mathrm{H} / \mathrm{C}$ & & \\
\hline & & & $\mathrm{g}$ & & 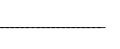 & & & & $\mathrm{g} \mathrm{kg}^{-1}$ \\
\hline \multirow[t]{2}{*}{$\mathrm{SD}$} & $\mathrm{AH}$ & $491(0,7)$ & $45(0,7)$ & 426 & $38(3,5)$ & 0,7 & 1,1 & 12,9 & 16 \\
\hline & $\mathrm{HU}$ & $382(1,4)$ & $42(2,8)$ & 550 & $26(2,8)$ & 1,1 & 1,3 & 14,7 & 540 \\
\hline \multirow[t]{2}{*}{$\mathrm{PC}$} & $\mathrm{AH}$ & $505(2,8)$ & $44(1,4)$ & 414 & $37(0,7)$ & 0,6 & 1,0 & 13,6 & 22 \\
\hline & $\mathrm{HU}$ & $409(1,4)$ & $58(2,1)$ & 512 & $21(2,1)$ & 0,9 & 1,7 & 19,5 & 620 \\
\hline
\end{tabular}

(1) Base livre de cinzas. ${ }^{(2)}$ Valores entre parêntese referem-se ao erro-padrão da média. ${ }^{(3)}$ Calculado por diferença.

\section{Sorção de acetochlor em amostra de solo}

Para ambos os tratamentos, a regressão linear entre quantidade sorvida e concentração em equilíbrio de acetochlor mostrou-se estatisticamente significativa a $1 \%$, obtendo-se coeficiente de determinação de 0,90 e 0,92 (Figura 1a). A capacidade sortiva de acetochlor em amostra de solo foi superior na semeadura direta em comparação à do preparo convencional, resultando em coeficientes de partição $K_{d}$ de 1,22 $\pm 0,11$ e 0,76 $\pm 0,08 \mathrm{~L} \mathrm{~kg}^{-1}$, respectivamente.

Considerando uma situação similar à dose aplicada em campo (concentração do herbicida na solução adicionada $=4,2 \mathrm{mg} \mathrm{L}^{-1}$ ), foi calculado o índice $\mathrm{K}_{\mathrm{d}}$, considerando os primeiros quatro pontos da isoterma (concentração inicial $=15 \mathrm{mg} \mathrm{L}^{-1}$ ). Os valores obtidos foram de $1,36 \pm 0,07 \mathrm{~L} \mathrm{~kg}^{-1}(\mathrm{r}=0,99)$, para a semeadura direta, e de $1,10 \pm 0,32 \mathrm{~L} \mathrm{~kg}^{-1}(\mathrm{r}=$ 0,93), para o plantio convencional, não indicando diferença entre os sistemas de manejo.

É possível que o preenchimento dos sítios de alta afinidade ocorra de maneira similar nos dois sistemas de manejo em virtude da elevada disponibilidade destes sítios relativamente à reduzida concentração de acetochlor. A diminuição de $K_{d}$ com o aumento da concentração de acetochlor adicionado, verificada nos presente estudo, foi reportada anteriormente em testes de sorção com um solo de textura média e teor de $\mathrm{C}$ orgânico de $57 \mathrm{~g} \mathrm{~kg}^{-1}$ ( Taylor et al., 2004). O comportamento sortivo não-linear foi atribuído pelos autores à competição entre co-solutos e interações específicas entre sorbato e os grupos ativos da MO, que se alterariam com o preenchimento gradual dos sítios sortivos.

Entretanto, considerando que aplicações de herbicidas em áreas agrícolas são anuais, a determinação do $K_{d}$ em intervalo maior de concentração é justificável para a avaliação da sorção de acetochlor em áreas de aplicações periódicas, em que o efeito re- sidual pode manifestar-se. Adicionalmente, deve-se ressaltar que, para a determinação de $\mathrm{K}_{\mathrm{d}}$ em laboratório, a suspensão é agitada por $24 \mathrm{~h}$, diferindo das condições verificadas em campo para o contato herbicida-solo. Este fato limita a comparação direta de resultados obtidos em laboratório com resultados de experimentos em campo.

Estudos anteriores, relativos à sorção de herbicidas metolachlor e atrazina, indicaram que a semeadura direta, ao incrementar o teor de MO na superfície do solo, aumentou a quantidade sorvida do composto (Pusino et al., 1992; Levanon et al., 1993). A contribuição da MO para a sorção do acetoclhor observada nos dois sistemas de manejo do solo no presente estudo foi avaliada pela comparação dos valores de $\mathrm{K}_{\mathrm{OC}}$, que foram de $86 \pm$ $8 \mathrm{~L} \mathrm{~kg}^{-1} \mathrm{C}$, para o preparo convencional, e de $116 \pm$ $10 \mathrm{~L} \mathrm{~kg}^{-1} \mathrm{C}$, para a semeadura direta. Este resultado mostra que a maior capacidade de sorção do acetochlor, manifestada na semeadura direta, não se deve apenas ao teor de $\mathrm{C}$ orgânico mais elevado neste sistema de manejo (Quadro 2). Diferentes características químicas e estruturais da MO do solo nos dois sistemas de manejo (tais como: proporção dos grupos funcionais e arranjo organomineral) podem ser também responsáveis por este comportamento. Os valores de $\mathrm{pH}$ da solução que continha o herbicida, antes e depois da sorção, foram semelhantes nos dois tratamentos (aproximadamente 5,5), bem como a área superficial específica das amostras (Quadro 1), razão pela qual tais fatores não estão relacionados com as diferenças de $\mathrm{K}_{\mathrm{OC}}$.

Analogamente ao presente estudo, Ferri et al. (2002) obtiveram um valor de $\mathrm{K}_{\mathrm{d}} 1,6$ vez superior em solo sob semeadura direta em comparação ao observado no preparo convencional, para a sorção de acetochlor em amostras de um experimento de sete anos no mesmo tipo de solo, onde foi empregada a rotação aveia preta e soja. Os valores de $\mathrm{K}_{\mathrm{d}}$ e $\mathrm{K}_{\mathrm{OC}}$ para ambos os tratamentos naquele estudo foram superiores $\left(2,2\right.$ e 1,4 vezes, para $\mathrm{K}_{\mathrm{d}}$ e $\mathrm{K}_{\mathrm{OC}}$, 



Figura 1. Relação entre concentração de equilíbrio (Ceq) e quantidade sorvida de acetochlor (Qs) em amostras de solo (a), de ácidos húmicos (b) e de huminas (c) de Argissolo Vermelho submetido à semeadura direta (SD) e ao preparo convencional (PC).

respectivamente) aos observados no presente estudo. Este fato deveu-se, provavelmente, ao diferente teor de $\mathrm{C}$ orgânico do solo e aos diferentes tempos do experimento e rotação de culturas empregados no presente estudo.

Os valores de $K_{d}$ e $K_{O C}$ observados neste estudo estão dentro dos limites verificados anteriormente para sorção de herbicidas do grupo químico dos cloroacetamidas em solos: 0,27 e 1,84 L kg-1, para $\mathrm{K}_{\mathrm{d}}$, e 9,5 e $147,9 \mathrm{~L} \mathrm{~kg}^{-1} \mathrm{C}$, para $\mathrm{K}_{\mathrm{OC}}$, de metolachlor (Crisanto et al., 1995), de 1,67 e 2,75 L kg-1, para $\mathrm{K}_{\mathrm{d}}$, e 126 e $166 \mathrm{~L} \mathrm{~kg}^{-1} \mathrm{C}$, para $\mathrm{K}_{\mathrm{OC}}$, de acetochlor (Ferri et al., 2002).

\section{Sorção de acetochlor em ácidos húmicos e huminas}

Analogamente ao verificado nas isotermas obtidas com as amostras de solo, a regressão linear calculada a partir dos dados experimentais para a sorção em ácidos húmicos e em huminas resultou estatisticamente significativa a $1 \%$, obtendo-se coeficientes de determinação de 0,87 a 0,95 (Figura 1b e 1c). Neste tipo de sorvente, o comportamento sortivo não se diferenciou com o manejo do solo (Figura 1b): o valor de $\mathrm{K}_{\mathrm{d}}$ do acetochlor em ácidos húmicos de preparo convencional $\left(178 \pm 18,9 \mathrm{~L} \mathrm{~kg}^{-1}\right)$ foi semelhante ao valor obtido para ácidos húmicos de amostra de semeadura direta $\left(158 \pm 14,6 \mathrm{~L} \mathrm{~kg}^{-1}\right)$. Os valores obtidos neste trabalho assemelham-se aos observados por Senesi et al. (1994) que verificaram valores de $\mathrm{K}_{\mathrm{d}}$ entre 116 e $170 \mathrm{~L} \mathrm{~kg}^{-1}$ para sorção de alachlor em ácidos húmicos de diferentes origens. Considerando concentrações de atrazina em equilíbrio até $15 \mathrm{mg} \mathrm{L}^{-1}$, índice $\mathrm{K}_{\mathrm{d}}$ calculado $\left(\mathrm{R}^{2}=\right.$ $0,88, \mathrm{P}<0,01$ ) decresce a 83,4 \pm 32 e 85,3 $\pm 33 \mathrm{~L} \mathrm{~kg}^{-1}$, para ácidos húmicos de semeadura direta e de preparo convencional, respectivamente. O aumento da afinidade entre sorbato e sorvente com o aumento da concentração de acetochlor adicionado ao sistema indica um aumento da disponibilidade dos sítios sortivos, conforme progride a sorção (Sposito, 1989). Comportamento similar foi verificado na sorção de atrazina em Cambissolo Húmico (Gomes et al., 2002), o qual foi atribuído ao efeito cooperativo entre moléculas de herbicida sorvidas.

$\mathrm{O}$ coeficiente $\mathrm{K}_{\mathrm{OC}}$ calculado, considerando todos os dados experimentais para o herbicida sorvido em ácidos húmicos de preparo convencional, foi de 352 $\pm 37 \mathrm{~L} \mathrm{~kg}^{-1} \mathrm{C}$ e para ácidos húmicos de semeadura direta foi de $321 \pm 30 \mathrm{~L} \mathrm{~kg}^{-1} \mathrm{C}$. O comportamento sortivo similar não é surpreendente em vista da semelhança da composição química dos dois sorventes (Quadro 2).

Nas huminas, o coeficiente de distribuição $K_{d}$ foi de $1.028 \pm 121 \mathrm{~L} \mathrm{~kg}^{-1}$ e de $1.183 \pm 180 \mathrm{~L} \mathrm{~kg}^{-1}$, na semeadura direta e preparo convencional, respectivamente. Contrariamente ao observado para os ácidos húmicos, não houve diminuição de $\mathrm{K}_{\mathrm{d}}$, considerando apenas concentrações baixas de acetochlor $\left(\mathrm{C}_{\mathrm{i}} \leq 15 \mathrm{mg} \mathrm{L}^{-1}\right): 1.260 \pm 300 \mathrm{~L} \mathrm{~kg}^{-1}$, para semeadura direta, e $1.230 \pm 300 \mathrm{~L} \mathrm{~kg}^{-1}$, para preparo convencional.

A similaridade do comportamento sortivo entre os dois tipos de huminas manteve-se no índice $\mathrm{K}_{\mathrm{OC}}$ : $2.691 \pm 317 \mathrm{~L} \mathrm{~kg}^{-1} \mathrm{C}$, para semeadura direta, e 2.892 $\pm 440 \mathrm{~L} \mathrm{~kg}^{-1}$, para o preparo convencional. 
Os valores de $\mathrm{K}_{\mathrm{d}}$ e $\mathrm{K}_{\mathrm{OC}}$ obtidos das isoterma com huminas foram seis a nove vezes superiores aos observados para os ácidos húmicos. O maior grau de oxidação (expresso pela razão $\mathrm{O} / \mathrm{C}$ ) e o maior caráter alifático das huminas em comparação aos ácidos húmicos (Quadro 2) poderiam propiciar a maior sorção de acetochlor nas huminas. Os grupos oxigenados mais abundantes nesta fração contribuiriam para as reações da MO com o acetochlor por meio de pontes de $\mathrm{H}$, assim como a maior presença de grupos alifáticos estimularia a retenção do produto por meio de interações hidrofóbicas. Chefetz et al. (2000) verificaram para sorção de compostos hidrofóbicos em huminas valores de $\mathrm{K}_{\mathrm{OC}} 10$ vezes superiores aos obtidos em ácidos húmicos. Naquele trabalho, a maior proporção de grupamentos alifáticos em huminas, determinada por espectroscopia de $\mathrm{RMN}{ }^{13} \mathrm{C}$, e a relação direta entre $K_{O C}$ e grau de alifaticidade obtida para os diferentes sorventes comprovaram a importância de estruturas alifáticas no processo de sorção.

Todavia, no presente estudo, as diferenças de composição química, determinadas por análise elementar, entre ácidos húmicos e huminas, não foram tão acentuadas como no trabalho de Chefetz et al. (2000), e alterações da capacidade sortiva decorrentes do processo de extração das substâncias húmicas devem ser consideradas.

As frações huminas após tratamento com solução de HF apresentaram elevado teor de fluoretos $\mathrm{Al}_{2}\left(\mathrm{SiF}_{6}\right)_{3}, \mathrm{Na}_{3} \mathrm{AlF}_{6}$ e NaF, identificados por DRX, os quais precipitaram durante o processo de solubilização de silicatos. A presença de fluoretos que revelam a ligação F-metal ( $\mathrm{Al}$ e $\mathrm{Si}$ ) em huminas fracionadas com solução de $\mathrm{NaOH}$ e concentradas com solução de HF foi também observada por Gomes (2002) por espectroscopia de infra-vermelho. Considerando a elevada eletronegatividade do $\mathrm{F}$, infere-se que a alta sorção do acetochlor, verificada nas frações huminas, é decorrente de sua interação não só com a MO, mas, também com os minerais existentes nas huminas fracionadas.

\section{Avaliação do comportamento dos três sorventes}

Os valores de $\mathrm{K}_{\mathrm{d}}$ e $\mathrm{K}_{\mathrm{OC}}$ mais elevados nos ácidos húmicos e huminas, quando comparados aos obtidos na amostra de solo, mostram que as frações orgânicas do solo foram os principais sorventes deste herbicida, conforme verificado por outros autores para o herbicida alachlor (Peter \& Weber, 1985; Senesi et al., 1994). A contribuição de cada fração para a sorção total do solo pode ser estimada multiplicando-se o valor de $\mathrm{K}_{\mathrm{OC}}$ da fração húmica pela proporção do $\mathrm{C}$ orgânico do solo contido nesta fração. Para a semeadura direta, o valor do $\mathrm{K}_{\mathrm{OC}}$ proporcional foi de $64,2 \mathrm{~L} \mathrm{~kg}^{-1} \mathrm{C}$, para ácidos húmicos, e de $1.292 \mathrm{~L} \mathrm{~kg}^{-1} \mathrm{C}$, para huminas. Para o preparo convencional, foram obtidos $84,5 \mathrm{~L} \mathrm{~kg}^{-1} \mathrm{C} \mathrm{e}$ $1.388 \mathrm{~L} \mathrm{~kg}^{-1} \mathrm{C}$, para ácidos húmicos e huminas, respectivamente. Em ambos os tratamentos, a soma dos valores de $\mathrm{K}_{\mathrm{OC}}$ proporcional das frações húmicas estudadas supera de 11 a 17 vezes (semeadura direta e preparo convencional, respectivamente) o valor de $\mathrm{K}_{\mathrm{OC}}$ do solo. Cumpre lembrar que a fração de ácidos fúlvicos, que não foi contemplada nesta estimativa, também pode contribuir para a sorção de acetochlor no solo.

Possivelmente, o isolamento dos sorventes orgânicos do solo leva a uma alteração de sua capacidade sortiva e de seu comportamento conforme o manejo. Esta alteração provavelmente decorre do fato de serem as interações organominerais e os arranjos micromorfológicos afetados pelos tratamentos químicos, alterando a reatividade do sorvente. Eventuais alterações na composição química dos sorventes, decorrentes dos tratamentos de extração das substâncias húmicas, que afetariam seu comportamento sortivo, também devem ser consideradas.

\section{CONCLUSÕES}

1. A capacidade sortiva de acetochlor foi superior em amostra de solo sob semeadura direta em relação ao preparo convencional. Este comportamento foi explicado apenas parcialmente pelo maior teor de $\mathrm{C}$ no tratamento da amostra de semeadura direta.

2. Quando isoladas, as huminas e ácidos húmicos apresentaram capacidade de sorver acetochlor mais elevada do que quando contidas no solo.

3. As huminas apresentaram maior capacidade de sorver acetochlor do que os ácidos húmicos.

\section{AGRADECIMENTOS}

À CAPES e ao CNPq, pelas bolsas de pósgraduação concedidas aos primeiro e segundo autores; à companhia Monsanto do Brasil, pelo fornecimento do herbicida.

\section{LITERATURA CITADA}

ARSHAD, M.A.; SCHNITZER, M.; ANGERS, D.A. \& RIPMEESTER, J.A. Effects of till vs no-till on the quality of soil organic matter. Soil Biol. Biochem., 22:595-599, 1990.

BAYER, C. \& BERTOL, I. Características químicas de um Cambissolo Húmico afetadas por sistemas de preparo, com ênfase à matéria orgânica. R. Bras. Ci. Solo, 23:687-694, 1999. 
BAYER, C.; MARTIN-NETO, L.; MIELNICZUK, J.; SAAB, S.C.; MILORI, D. \& BAGNATO, V.S. Tillage and cropping system effects on soil humic acid characteristics as determined by electron spin resonance and fluorescence spectroscopies. Geoderma, 105:81-92, 2002.

CHEFETZ, B.; DESHMUKH, A.P.; HATChER, P.G. \& GUTHRIE, E.A. Pyrene sorption by natural organic matter. Environ. Sci. Technol., 34:2925-2930, 2000.

CRISANTO, T.; SANCHEZ-CAMAZANO, M.; ARIENZO, M. \& SANCHEZ-MARTIN, M.J. Adsorption and mobility of metolachlor in surface horizons of soil with low organic matter content. Sci. Total Environ., 166:69-76, 1995.

CZAPAR, G.F.; KANVAR, R.S. \& FAWCETT, R. Herbicide and trace movement to field drainage tiles under simulated rainfall conditions. Soil Till. Res., 30:19-32, 1994.

DICK, D.P.; GOMES, J. \& ROSINHA, P.B. Caracterização de substâncias húmicas extraídas de solos e de lodo orgânico. R. Bras. Ci. Solo, 22: 603-611, 1998.

DICK, D.P.; SANTOS, J.H.Z. \& FERRANTI, E.M. Chemical characterization and infrared spectroscopy of soil organic matter from two southern Brazilian soils. R. Bras. Ci. Solo, 27: 29-39, 2003.

DOANE, T.A.; DEVÊVRE, O.C. \& HORWÁTH, W.R. Short-term soil carbon dynamics of humic fractions in low-input and organic cropping systems. Geoderma, 114:319-331, 2003.

EMPINOTTI, V.L. Conteúdo e qualidade da matéria orgânica em sistemas de manejo e classes de solo. Porto Alegre, Universidade Federal do Rio Grande do Sul, 1999. 101p. (Tese de Mestrado)

EMPRESA BRASILEIRA DE PESQUISA AGROPECUÁRIA EMBRAPA. Serviço Nacional de Pesquisa de Solos. Sistema Brasileiro de Classificação de Solo. Rio de Janeiro, 1999. 412p.

FERRI, M.V.W.; VIDAL, R.A.; GOMES, J.; DICK, D.P. \& SOUZA, R.F. Atividade do herbicida acetochlor em solo submetido à semeadura direta e ao preparo convencional. Pesq. Agropec. Bras., 37:1697-1703, 2002.

GOMES, J. Sorção dos herbicidas atrazina e acetochlor em substâncias húmicas de diferentes origens. Porto Alegre, Universidade Federal do Rio Grande do Sul, 2002. 97p. (Tese de Mestrado)

GOMES, J.; DICK, D.P. \& SOUZA, R.F. Sorção de atrazina em Cambissolo Húmico do Rio Grande do Sul sob vegetação nativa. R. Bras. Ci. Solo, 26:521-528, 2002.

HERWIG, U.; KLUMPP, E.; NARRES, H.D. \& MILAN, S.J. Physicochemical interactions between atrazine and clay minerals. Appl. Clay Sci., 18: 201-222, 2001.

KILE, D.E.; CHIOU, C.T. \& ZHOU, H. Partition of nonpolar organic pollutants from water to soil and sediment organic matters. Environ. Sci. Technol., 29:1401-1406, 1995.

KUCKUK, R.; HILL. W.; NOLTE. J. \& DAVIES, A.N. Preliminary investigations into the interactions of herbicides with aqueous humic substances. Pestic. Sci., 51:450-454, 1997.
LEE, D. \& FARMER, W.J. Dissolved organic matter interaction with napropamide and four other nonionic pesticides. J. Environ. Qual., 18:468-474, 1989.

LEVANON, D.; CODLING, E.E.; MEISSINGER, J.J. \& STARR, J.L. Mobility of agrochemicals through soil from two tillage systems. J. Environ. Qual., 22:155-161, 1993.

NAKAMAE, I.J., ed. Agrianual 2003:Anuário de agricultura brasileira. São Paulo: OESP Gráfica, 2002. 544p.

PETER, C.J. \& WEBER, B.J. Adsorption, mobility and efficacy of alachlor and acetochlor as influenced by soil properties. Weed Sci., 33:874-881, 1985.

PUSINO, A.; LIU,W. \& GESSA, C. Influence of organic matter and its clay complexes on metolachlor sorption on soil. Pestic. Sci., 36:283-286, 1992.

QUIRK, J.P. Significance of surface areas calculated from water vapour sorption isotherms by the use of BET equation. Soil Sci., 80:423-430, 1955.

RUTHERFORD, D.W.; CHIOU, C.T. \& KILE, D.E. Influence of soil organic matter composition on the partition of organic compounds. Environ. Sci. Technol., 26:336-340, 1992.

SCHEUNERT, I.; MANSOUR, M. \& ANDREUX, F. Binding of organic pollutants to soil organic matter. Intern. J. Environ. Anal. Chem., 46:189-199, 1992.

SCHMIDT, W.W.I.; KNICKER, H.; HATCHER, P.G. \& KÖGELKNABNER, I. Improvement of ${ }^{13} \mathrm{C}$ and ${ }^{15} \mathrm{~N}$ CPMAS NMR spectra of bulk soils, particle size fractions and organic material by treatment with $10 \%$ hydrofluoric acid. Eur. J. Soil Sci., 48:319-328, 1997.

SENESI, N.; BRUNETTI, G.; LA CAVA, P. \& MIANO, T.M. Adsorption of alachlor by humic acids from sewage sludge and amended and non-amended soils. Soil Sci.,157:176-184, 1994.

STEARMAN, G.K.; LEWIS, R.J.; TORTEROLLI, L.J. \& TYLER, D.D. Herbicides reactivity of soil organic matter fractions in no-tilled and tilled cotton. Soil Sci. Soc.Am. J., 53:16901694, 1989.

SPOSITO, G. The chemistry of soils . New York, Oxford University Press, 1989. 223p.

STEVENSON, F.J. Organic matter reactions involving herbicides in soil. J. Environ. Qual., 1:333-343, 1972.

STEVENSON, F.J. Humus chemistry: Genesis, composition, reactions. New York, John Wiley, 1994. 443p.

SWIFT, R.S. Organic matter characterization. In: SPARKS, D.L. ??DEMAIS EDITORES??, eds. Methods of soil analysis. Part 3. Chemical methods. Madison, Soil science Society of America, 1996. p.1011-1065.

TAYLOR, J.P., MILLS, M.S. \& BURNS, R.G. Sorption and desorption behaviour of acetochlor in surface, subsurface and size-fractionated soil. Eur. J. Soil Sci., 55:671-679, 2004.

TEDESCO, M.J.; GIANELLO, C.; VOLKWEISS, S.J. \& BISSANI, C.A. Análises de solo, plantas e outros materiais. Porto Alegre, Universidade Federal do Rio Grande do Sul, 1995. 174 p. (Boletim Técnico de Solos, 5) 
TORRENTS, A.; JAYASUNDERA, S. \& SCHMIDT, W.J. Influence of the polarity of organic matter on the sorption of acetamide pesticides. J. Agric. Food Chem., 45:3320-3325, 1997.

VIDAL, A.R. \& FLECK, N.G. Inibidores de crescimento da parte aérea. In: VIDAL, A.R. \& MEROTTO JR., A., eds. Herbicidologia. Porto Alegre, Universidade Federal do Rio Grande do Sul, 2001.152p.
WEBER, J.B. \& PETER, C.J. Adsorption, bioactivity and evaluation of soil tests for alachlor, acetochlor and metolachlor. Weed Sci., 30:14-20, 1982.

WOOD, L.S.; SCOTT, H.D.; MARX, D.B. \& LAVY, T.L Variability in sorption coefficients of metolachlor on a Captina silt loam. J. Environ. Qual., 16:251-256, 1987. 